\section{An automatic frequency-sweeping SQUID susceptometer}

\author{
J A Overweg, H J M ter Brake, J Flokstra and G J Gerritsma \\ Twente University of Technology, PO Box 217, $7500 \mathrm{AE}$ \\ Enschede. The Netherlands
}

Received 21 March 1983

\begin{abstract}
A new, sQuid-based, measuring system has been developed for the investigation of the dynamic properties of magnetic materials. Its main advantages, compared to conventional mutual inductance systems, are its high sensitivity and its nearly frequency independent response, down to extremely low frequencies. With the sQuid system it is now possible to measure directly and rapidly the frequency dependence of the dynamic susceptibility of weakly magnetic materials in the range from $0.002 \mathrm{~Hz}$ to $5 \mathrm{kHz}$ at a fixed value of a constant background field of up to $5 \mathrm{~T}$.

The frequency-swept operation allows experiments which are beyond the capabilities of conventional systems, for example the investigation of thermally isolated samples or the study of the dynamics at magnetic phase transitions in cases where the magnetic properties change very rapidly in a small field interval.
\end{abstract}

\section{Introduction}

Dynamic properties of magnetic materials are often studied by means of a mutual inductance method, in which the response of a sample to a harmonically varying magnetic field is measured for different values of the frequency of this AC field. To this end a sample of the material under investigation is placed inside a coil system (figure 1), consisting of a primary coil. which generates a small AC field, and an astatic secondary coil (two equally large but oppositely wound sections), which picks up the response of the sample. This secondary coil is connected to a detection system. For the generation of a quasi-constant background field in the experiments the system of sensing coils is located inside the bore of a larger magnet coil.

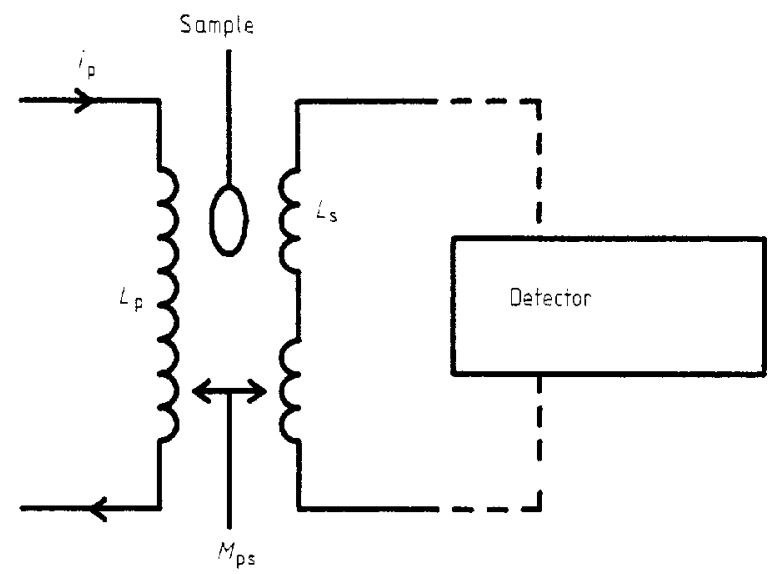

Figure 1. Principle of mutual inductance technique.
In conventional mutual inductance systems the detector is usually a sensitive tuned lock-in amplifier. which amplifies the voltage induced in the secondary coil and determines its in-phase and quadrature components at the frequency of the reference. By introducing a SQuID as a detector for the secondary signal the sensitivity of the system can be improved greatly compared to the classical inductive technique, especially at low frequencies. Furthermore, the nearly flat frequency response of the SQCID makes it now possible to do successive measurements at different frequencies in a short time. The lock-in amplifier can then be used in the flat mode.

In this paper we describe the sQuID-based frequency sweeping susceptometer we developed for our magnetic relaxation measurements (Overweg et al 1980). After the establishment of the shortcomings of the classical mutual inductance technique $(\$ 2)$ we pay attention to some design criteria for a SQUiD susceptometer $(\S 3)$. The properties of the RF SQUID, the feedback circuitry and the flux transformer, relevant for the operation of the system, are described in $\S \S 4.5$ and 6 . The complete experimental set-up is presented in $\S 7$ and finally we demonstrate the frequency-sweeping susceptometer for measurements on some magnetic materials $(\$ 8)$.

The measurements were performed with the aid of a minicomputer which controls the oscillator for the primary field and processes the output signal. When the sQuid was used in combination with a lock-in amplifier in flat mode, the measurements were restricted to the frequency range from $10 \mathrm{~Hz}$ to about $5 \mathrm{kHz}$. For the low-frequency range we developed a digital phase-sensitive detector and were able to extend the frequency range to about $0.002 \mathrm{~Hz}$. The upper frequency limit of the digital PSD is about $2 \mathrm{kHz}$. The digital PSD and the control of the measuring process have been described in a separate paper (Gerritsma et al 1983).

With our system it is for the first time possible to perform a completely automatic measurement of the frequency dependence of the dynamic susceptibility $\chi=\chi^{\prime}-\mathrm{i} \chi^{\prime \prime}$ of magnetic materials.

\section{Shortcomings of the classical mutual inductance technique}

In the classical mutual inductance method the voltage $V_{\mathrm{s}}$ induced in the secondary coil is detected (see e.g. Van de Klundert et al (1975) and Flokstra et al (1980)). If the output voltage is analysed with a two-phase lock-in amplifier the quadrature output is proportional to $\chi^{\prime}$. whereas the in-phase signal is a measure for $\chi^{\prime \prime}$ (provided the coil system is perfectly balanced). The induced voltage is proportional to the number of turns of the secondary coil and to the frequency of the AC field. At low frequencies a large number of turns and a highly selective amplifier are usually necessary to achieve an acceptable signalto-noise ratio. We shall demonstrate this in the next example.

If a sample of susceptibility $\chi$ is placed in one of the secondary sections of the sensing mutual inductor the total magnetic flux through this secondary coil due to a current $i_{\mathrm{p}}$ in the primary coil is

$$
\varphi_{\mathrm{s}}=M_{p s} i_{p} f \chi+\Delta M_{p s} i_{p} .
$$

Here the first term represents the signal from the sample. $f$ being a geometry-dependent filling factor. The second term is the contribution from the imbalance of the coil system (a small difference $\Delta M_{\mathrm{ps}}$ between the mutual inductances $M_{\mathrm{ps}}(1)$ and $M_{\mathrm{ps}}(2)$ between the primary coil and the two secondary sections is inevitable).

In most practical cases the diameter of the sample is relatively small compared to the diameter of the secondary coil. For an estimate of the magnitude of $\varphi_{s}$ the sample can then be regarded as a dipole of moment $m=\chi V V H_{p}$ ( $V$ is the volume of 
the sample and $H_{\mathrm{p}}$ the amplitude of the alternating magnetic field). If this dipole is located in the centre of a secondary section of $N_{\mathrm{s}}$ turts of radius $r_{s}$ the flux $\Delta \varphi_{\mathrm{s}}$ due to the presence of the sample becomes

$$
\Delta \varphi_{\mathrm{s}}=N_{\mathrm{s}} \mu_{0} \chi V H_{\mathrm{p}} / 2 r_{\mathrm{s}} .
$$

A typical numerical example: if $r_{\mathrm{s}}=5 \mathrm{~mm}, \mu_{0} H_{\mathrm{p}}=0.1 \mathrm{mT}(1 \mathrm{G})$, $\chi=10^{-2}$ and $V=10^{-7} \mathrm{~m}^{3}$ the flux picked up by the secondary coil is $\Delta \varphi_{\mathrm{s}}=10^{-11} N_{\mathrm{s}} \mathrm{Wb}$. In the mutual inductance technique the time derivative of $\Delta \varphi_{\mathrm{s}}$ is measured and in this example the induced voltage is only $6 \mathrm{nV}$ for a coil of 100 turns at a frequency of $1 \mathrm{~Hz}$. This low value cannot be measured with a lock-in amplifier in experimental circumstances.

The mutual inductance technique can be applied quite satisfactorily for the investigation of the field and temperature dependence of the susceptibility, when magnetic relaxation effects are not present. The measuring frequency can then be fixed at a suitable high value, at which the induced voltage is large enough to be detected with sufficient accuracy. The field dependence of the susceptibility can easily be studied by sweeping the magnetic field.

When, however, the frequency dependence of the susceptibility becomes of interest, this measuring technique has serious limitations. Firstly, the low sensitivity of the inductive method at low frequencies (demonstrated above) makes it very difficult to investigate slow relaxation processes. The minimum operating frequency lies usually at about $1 \mathrm{~Hz}$. Secondly, it is not possible to perform a large number of measurements at different frequencies in a short time, let alone perform a continuous frequency sweep. In principle it is possible to correct afterwards for the increase of the output signal with frequency. The main obstacle for frequency-swept operation is the time consuming readjustment of the selective preamplifier which is necessary after each frequency change. At higher frequencies the stray capacitances in the sensing coils, which may be fairly large due to the required large number of turns, can cause unwanted phase shifts which have to be corrected for. A maximum measuring rate of one frequency point in approximately $3 \mathrm{~min}$ could be achieved with a typical inductance system used in our laboratory (Flokstra et al 1980).

Because of the problems involved with frequency changes, the frequency dependence of the susceptibility is usually investigated in the rather indirect way of making a number of field sweeps at different fixed frequencies. There are, however, a number of situations where this field sweep method cannot be used. One example is the investigation of relaxation effects near magnetic phase transitions, where the magnetic properties of the sample change abruptly in a small field interval (see $\$ 8$ ). Especially when the magnetic behaviour of the sample is strongly anisotropic it is very difficult to perform a number of consecutive field sweeps under exactly the same conditions. In order to correct for contributions due to the coil system itself each field sweep has to be repeated for an empty coil and the repositioning of the sample may lead to large errors in the susceptibility. Another example where a frequency-sweep possibility would be advantageous is the investigation of a sample covered with a thin helium film which provides a far better thermal stabilisation than a bath of liquid helium does (Overweg et al 1982). The thickness of such a film would be too seriously infuenced by the magnetocaloric effect of a field sweep. Similar problems arise when thermally isolated samples have to be investigated in order to obtain the intrinsic spin-lattice relaxation phenomena.

\section{Some design considerations for a SQUID susceptometer}

In order to obtain a susceptometer which operates down to much lower frequencies than a conventional inductive system and which allows frequency-swept operation, a system was developed in which SQUID detects changes in the flux picked up by the secondary coil.

For many years SQUIDs have been used in the investigation of the magnetic properties of solids (Giffard et al 1976 , Williamson and Kaufman 1981). In nearly all cases, however, the experimental arrangement is laid out for static measurements, with the highest achievable sensitivity (Cukauskas et al 1973, Cerdonio et al 1976, Hirschkoff et al 1971, Philo and Fairbank 1977). This usually involves stabilisation of the external magnetic field by means of one or more superconducting shields. Obviously, such a rigorous field stabilisation is not very suitable if the response of a sample to an oscillating field is to be studied.

For the investigation of the frequency dependence of dynamic susceptibilities it is desirable that the detection system has a flat response over a large frequency range. In order to achieve this both the response of the SQuID itself and that of the system of sensing coils have to be considered (described in the next sections). In particular the SQUID needs attention, since most commercially available sQuID systems are optimised for low-frequency stability rather than good high-frequency performance. The AC-SQUID susceptometer does not necessarily have to be optimised for the highest possible sensitivity, so that the extensive magnetic shielding and field stabilisation usually found in static magnetometers can be omitted.

\section{The RF-SQUID}

As a detector in the new susceptometer a commercially available RF-SQUID system (SHE-330 with TSQ sensor) is used. Some of its important properties, mainly connected with its frequency response, will be described in the following.

An RF-SQLID is in principle a ring-shaped superconducting structure which is closed in itself by a single Josephson junction. The device measures the magnetic flux through the ring, which is in nearly all applications generated by a small coil (the signal coil) located inside the SQUID ring. The SQUID is inductively coupled to an $L C$ tank circuit driven to resonance by an $\mathrm{RF}$ oscillator (in the SHE system at $19 \mathrm{MHz}$ ). At a suitably selected value of the RF current the amplitude of the voltage across the tank circuit $V_{\mathrm{RF}}$ is modulated in the well known triangular way by the total magnetic flux $\varphi_{\mathrm{SQ}}$ coupled into the sQuid ring.

Important are the following properties of the triangular transfer function.

(i) The behaviour of $V_{\mathrm{RF}}$ is purely periodic in the applied flux $\varphi_{\mathrm{SQ}}$ with a periodicity of one flux quantum $\varphi_{0}=h / 2 e=$ $2.07 \times 10^{-15} \mathrm{~Wb}$. In principle this periodicity remains as long as the SQUID is superconducting and is, within this restriction, independent of the magnitude of the applied flux or the temperature.

(ii) Because of the perfect periodicity of the transfer function, $V_{\mathrm{RF}}$ is determined only by the difference between $\varphi_{\mathrm{SQ}}$ and the nearest integer number of flux quanta. This relative character of the measurement allows the detection of very small flux changes against a much larger constant background.

(iii) $V_{\mathrm{RF}}$ is determined by the instantaneous value of $\varphi_{\mathrm{SQ}}$, so that even quasi-stationary changes in the input signal can be detected.

(iv) The high-frequency properties of the RF part of the SQLID system are mainly determined by the bandwidth of the RF preamplifier which is $150 \mathrm{kHz}$ for the SHE $300 \mathrm{RF}$ head. The frequency response of the basic RF-SQUID is shown in figure 2 .

Because the basic RF-SQUID only operates linearly when the input flux varies between $n \varphi_{0}$ and $\left(n+\frac{1}{2}\right) \varphi_{0}$ the usefulness of this part of the system alone is rather limited. To overcome this problem, the SQUID is usually incorporated into a feedback loop (figure 3 ), in which any change $\delta \varphi_{\mathrm{SQ}}$ in the flux coupled into the 


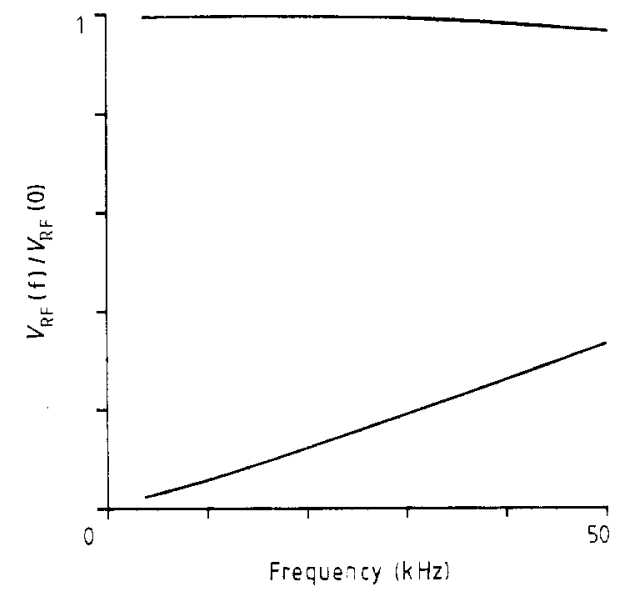

Figure 2. Response of bașic SQuID: upper curve, in-phase: lower curve, quadrature.

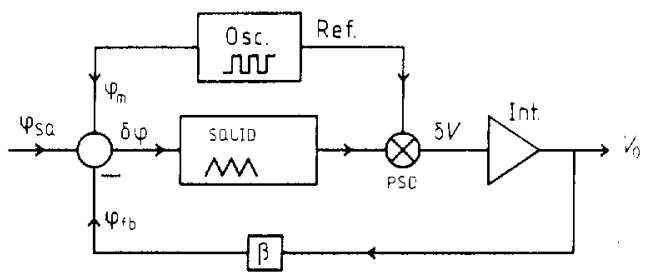

Figure 3. sQuID in feedback loop.

SQUID by the signal coil is counterbalanced by an equally large change of a feedback flux $\delta \varphi_{\mathrm{fb}}$. The direction of the flux change is obtained with a $50 \mathrm{kHz}$ block wave modulation with an amplitude of $\varphi_{0} / 4$. The system is thus locked to one of the minima in the triangular transfer function of the SQUID and the total flux in the sQuid changes by no more than a fraction of a flux quantum. In the (standard) internal feedback mode of the SHE-330 system the feedback signal is superimposed upon the RF signal and is coupled into the SQUID via the RF coil.

As long as the system remains locked on the same number of flux quanta, the voltage $V_{0}$, which generates the feedback flux, changes proportionately to the changes in $\varphi_{\mathrm{SQ}}$ and is therefore used as the output signal. The overall gain of the system, $\Delta V_{0} / \Delta \varphi_{\mathrm{SQ}}$ is now determined by the effective feedback resistance $R_{\mathrm{fb}}$ (the ratio of $V_{0}$ and the feedback current $i_{\mathrm{fb}}$ (in internal feedback mode through the RF coil)), and by the mutual inductance $M_{\mathrm{fb}}=\varphi_{\mathrm{rb}} / i_{\mathrm{fb}}$

$$
\frac{\Delta V_{\mathrm{o}}}{\Delta \varphi_{\mathrm{SQ}}}=\frac{R_{\mathrm{fo}}}{M_{\mathrm{fb}}}=\frac{1}{\beta} .
$$

The feedback factor, $\beta$, is independent of the frequency of the input signal (provided stray capacitances in the feedback circuit can be neglected). In the frequency range where the loop gain is sufficiently high the system will thus have a flat frequency response. The calibration is also insensitive to changes in the temperature of the SQUID or the adjustment of the RF current.

\section{Dynamic properties of the feedback loop}

For low-frequency signals the maximum change in $\varphi_{\mathrm{SQ}}$ which the feedback loop can follow is limited by clipping of the output voltage at $10 \mathrm{~V}$. In the SENS $\times 1$ mode this corresponds to a dynamic range of $500 \varphi_{0}$. When $V_{0}$ becomes too large the feedback loop has to be interrupted temporarily. After closing the loop again the system locks onto another minimum in the triangular transfer function, which is then taken as a new zero point for further measurements. This reset operation is performed automatically when $V_{0}$ exceeds a preset threshold voltage. The autoreset feature permits measurements of signals which are much larger than $500 \varphi_{0}$. This is, however, not possible at signal frequencies above a few hertz, as each autoreset takes approximately $50 \mathrm{~ms}$.

At frequencies above approximately $1 \mathrm{kHz}$ the integrator (see figure 3), which ensures stable operation of the feedback loop, reduces the loop gain so much that the changes in the input flux are no longer fully compensated. This results in a phase shift between $V_{0}$ and $\varphi_{\mathrm{SQ}}$. If the open loop gain between the signal coil of the SQuid and the output is equal to $G(\omega)$ the general expression for the output voltage as a function of $\omega$ becomes

$$
V_{0}(\omega)=\left[\varphi_{\mathrm{SQ}}(\omega)-\left(n+\frac{1}{2}\right) \varphi_{0}\right] \frac{G(\omega)}{1+G(\omega) \beta} .
$$

For simplicity $G(\omega)$ can be assumed to be purely integrating: $G(\omega)=G_{0} /(1+i \omega \tau)$. With this approximation the closed loop transfer function becomes

$$
\frac{\Delta V_{0}(\omega)}{\Delta \varphi_{\mathrm{SQ}}} \approx \frac{1}{\beta} \frac{1}{1+\mathrm{i} \omega \tau^{*}}
$$

where $\tau^{*} \approx \tau / \beta G_{0}$ is the effective time constant of the system. The magnitude of $G_{0}$ depends on the selected feedback mode (see SHE manual). In FAST mode the $-3 \mathrm{~dB}$ point lies at $20 \mathrm{kHz}$. The actual characteristics of the system differ somewhat from the simple first-order shape predicted by equation (5), owing to the higher-order behaviour of $G(\omega)$. An example of measured frequency characteristics is shown in figure 4 .

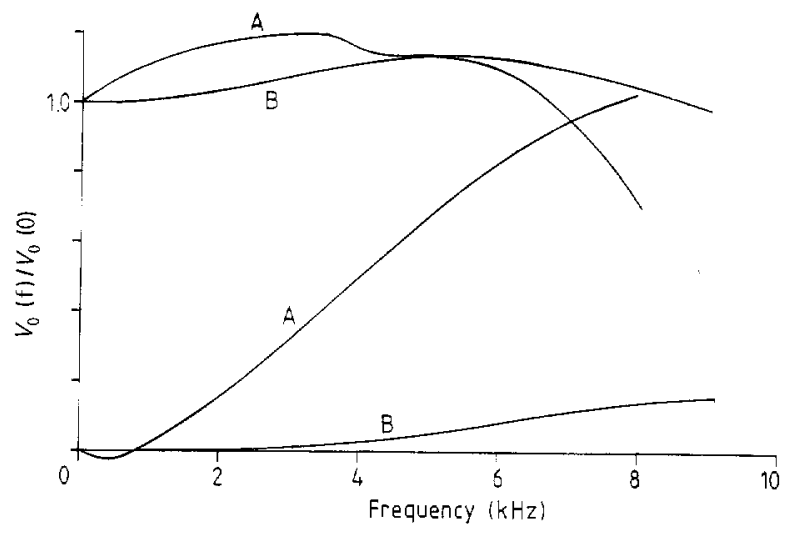

Figure 4. Frequency response of SQUID in feedback mode: A, Internal feedback (in-phase (upper) and quadrature (lower) component); B, external feedback (in-phase (upper) and quadrature (lower) component).

As a consequence of the fall off of the loop gain the system is slew-rate limited. The maximum amplitude of $\varphi_{\mathrm{SQ}}$ is determined by the speed at which the feedback circuit can track a change of $\varphi_{\mathrm{SQ}}$. Due to the reduction in loop gain, the magnitude of the error flux $\delta \varphi_{\mathrm{SQ}}$ becomes larger when $\mathrm{d} \varphi_{\mathrm{SQ}} / \mathrm{d} t$ increases. When, however, $\delta \varphi_{\mathrm{SQ}}$ exceeds $\varphi_{0} / 4$ the error voltage $\delta V$ ceases to be proportional to $\delta \varphi_{\mathrm{sQ}}$ and when the error flux becomes still larger the negative feedback will turn into positive feedback. As a consequence the system will loose its lock point 
and the linear relationship between $\varphi_{\mathrm{SQ}}$ and $V_{0}$ will be lost in a rather unpredictable way.

In order to ensure stable closed-loop operation the error flux $\delta \varphi_{\mathrm{SQ}}$ has to remain small compared to $\varphi_{0} / 4$, which implies for the externally applied flux

$$
\varphi_{\mathrm{SQ}}|=(1+G(\omega) \beta)| \delta \varphi_{\mathrm{SQ}} \mid<(1-G(\omega) \beta) \varphi_{0} / 4
$$

With the simplified first-order frequency dependence of $G(\omega)$ the maximum rate of change of a sinusoidal input signal $\varphi_{\mathrm{SQ}}$ becomes

$$
\left(\frac{\mathrm{d} \varphi_{\mathrm{SQ}}}{\mathrm{d} t}\right)_{\max } \approx \mathrm{i} \omega \frac{G_{0} \beta}{1+\mathrm{i} \omega \tau} \varphi_{0} / 4 \approx G_{0} \frac{\beta}{\tau} \varphi_{0} / 4
$$

(assuming, that $\omega \tau \geqslant 1$ ), which is independent of the frequency. The maximum slew-rate of the SHE- 330 system is $3 \times 10^{5} \varphi_{0} \mathrm{~s}^{-1}$ (in FAST mode). This implies for example at $1 \mathrm{kHz}$ a maximum signal amplitude of only $50 \varphi_{0}$.

The SHE-330 system has been designed for optimum stability at very low frequencies. In order to minimise the possibility of drift in the output signal due to changing thermoelectric voltages the feedback network incorporates an active voltage-to-current converter instead of a simple feedback resistor. This circuit is used in the frequency range below $800 \mathrm{~Hz}$ : at this frequency there is a gradual cross-over to another feedback path for higher frequencies. As a consequence of the existence of these separate DC and AC feedback paths, the standard system exhibits considerable phase shifts in the crossover frequency region (curve $\mathrm{A}$ in figure 4). A much better frequency response could be obtained, at the expense of a reduction in DC stability, by coupling the feedback signal into the SQUID via the signal coil rather than the RF coil. In this external feedback mode of operation the system has a nearly flat frequency characteristic up to approximately $5 \mathrm{kHz}$ (figure 4 , curve $B$ ).

We now have a very sensitive sensor, with a flat response over a reasonably large frequency range, especially at the lowfrequency end of the spectrum. The next section deals with the coupling between this sensor and the secondary coil of the sensing mutual inductor.

\section{The flux transformer}

If a SQUID is used as a detector in an AC susceptometer the measuring coil system will differ essentially from its usual design for a conventional mutual inductance bridge. In the latter case a large induced voltage is desired and the secondary coil has to have a large number of turns. The resistance of the coil is then relatively unimportant because of the usually high input impedance of the preamplifier of the detector system.

For a SQUID-based system a first requirement is that the secondary coil system is superconducting. Contrary to what one tends to expect, the maximum sensitivity is now obtained with a rather small number of secondary turns. The secondary coil, connected in series with the signal coil of the SQuID, forms a closed superconducting loop. which is usually called a flux transformer (figure 5).

If, for example, owing to the presence of a magnetic sample, the magnetic flux through the sensing coil changes by an amount $\Delta \varphi_{s}$, the current $i_{s}$ in the flux transformer will also change in such a way that the total magnetic flux surrounded by the superconducting loop remains constant at any time

$$
\Delta i_{s}=\Delta \varphi_{\mathrm{s}} / \Sigma L_{\mathrm{i}} \text {. }
$$

Here $\Sigma L_{f t}$ is the total self-inductance of the flux transformer. With a mutual inductance $M_{\mathrm{SQ}}$ between the signal coil and the SQUID the resulting flux change in the SQuid becomes

$$
\Delta \varphi_{\mathrm{SQ}}=\Delta \varphi_{\mathrm{s}} M_{\mathrm{SQ}} / \Sigma L_{\mathrm{f}}
$$

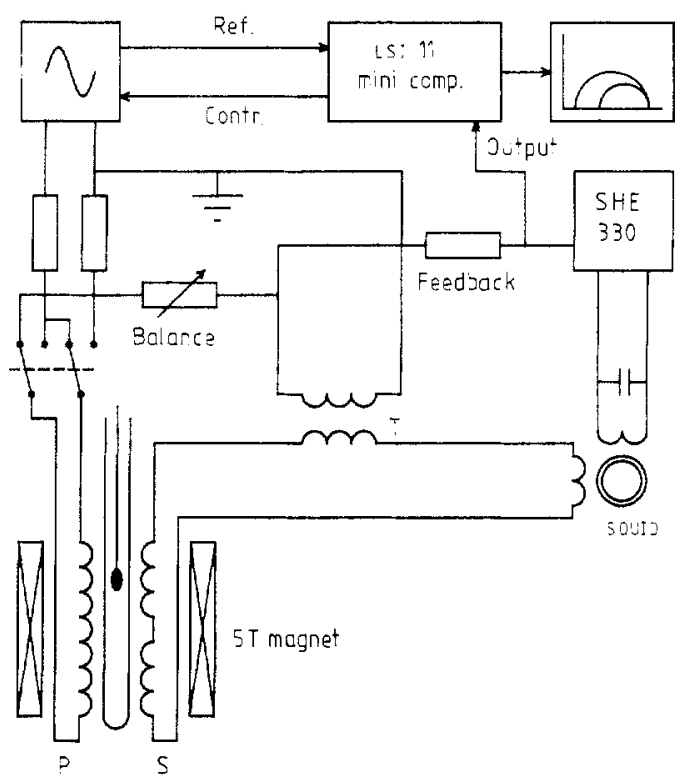

Figure 5. Diagram of the SQuid susceptometer

The flux-reduction factor $\Delta \varphi_{\mathrm{SQ}} / \Delta \varphi_{\mathrm{s}}$ is determined entirely by $M_{\mathrm{SQ}}$ and $\Sigma L_{\mathrm{ft}}$ and is, when capacitive and loss effects can be neglected, independent of the rate of change of $\varphi_{\mathrm{s}}$ (thus independent of frequency).

The two secondary astatic coil sections usually exhibit a certain imbalance and when performing accurate measurements, the effect of this imbalance has to be reduced with an additional balancing system. Because the entire fux transformer circuit has to be superconducting, it is impossible to use a variable mutual inductor at room temperature, in series with the sensing coils, as in a classical Hartshorn bridge. Instead, a second, fixed, mutual inductor, whose superconducting secondary coil is connected in series with the flux transformer, is used to compensate the imbalance (figure 5). The eurrent through the primary coil of this balancing inductor is derived from the current that generates the $\mathrm{AC}$ field by a simple resistive network. The reactive impedance of the coils can be neglected with respect to the ohmic resistances in the circuit. Because of the small number of turns of the coils capacitive effects can also be neglected, so that the balancing signal does not require a quadrature component with respect to the primary current.

The balancing mutual inductor is also used to couple the feedback signal into the flux transformer. Apart from the fact that the phase shifts due to the cross-over between the two feedback paths are thus avoided $(\S 5)$, this has the additional advantage that there is no net $\mathrm{AC}$ current induced in the flux transformer circuit. This minimises possible loss effects in the superconducting material of the coils.

The sensitivity of the system can be adapted to the expected signals through a suitable choice of the shape and the size of the sensing mutual inductor and the balance inductor. The mutual inductance $M_{\mathrm{SQ}}$ and self inductance $L_{\mathrm{SQ}}$ of the signal coil of the sQuID are fixed: $M_{\mathrm{SQ}}=21 \mathrm{nH}$ and $L_{\mathrm{SQ}}=2 \mu \mathrm{H}$. Assuming, that the self inductance $L_{\mathrm{s}}$ of one section of the secondary coil can be written as $L_{\mathrm{s}}=k_{\mathrm{s}} N_{\mathrm{s}}^{2}$ and neglecting the inductive coupling between the two sections of the secondary coil, the flux coupled into the SQUID becomes

$$
\Delta \varphi_{\mathrm{SQ}}=\frac{N_{\mathrm{s}} \mu_{0} \chi V H_{\mathrm{p}} M_{\mathrm{SQ}}}{2 r_{\mathrm{s}}\left(2 k_{\mathrm{s}} N_{\mathrm{s}}^{2}+L_{\mathrm{b}}+L_{\mathrm{SQ}}\right)} .
$$


Here $L_{\mathrm{b}}$ is the self inductance of the secondary coil of the balance mutual inductor. The inductance of the connecting wires is neglected. The maximum flux transfer is obtained by choosing a value of $N_{\mathrm{s}}$ such that $L_{\mathrm{s}}=\left(L_{\mathrm{b}}+L_{\mathrm{SQ}}\right) / 2$. However, because of the extremely high sensitivity of the SQUID it was for our applications not necessary to maximise the flux transfer. This was even unwanted, for in the experiments the signal may easily exceed the linear operating range of the SQUID. It is in principle always possible to avoid this situation by reducing the amplitude of the AC current through the primary coil. There is however, a practical limit to this procedure. As the magnetic moment of the sample is roughly proportional to $H / T$ the ratio between the $\mathrm{AC}$ component of the field $H_{\mathrm{p}}$ and its constant component $H_{0}$ has to remain large compared to the relative drifts in $H_{0}$ and $T$ during a single period of the AC field, otherwise these drifts become the limiting factors for the signal-to-noise ratio. Furthermore, the $\mathrm{AC}$ field must not become smaller than external disturbing magnetic fields which may enter the experimental set up. Also the effect of mechanical vibrations of the system may set a limit to the reduction of the primary current.

\section{Technical data of the experimental set-up}

In order to minimise the inductive coupling between the primary coil and the superconducting magnet, the primary coil has been made astatically. It consists of two concentric single-layer coils of $0.1 \mathrm{~mm}$ copper wire with a length of $60 \mathrm{~mm}$. The inner coil has 300 turns of $19.1 \mathrm{~mm}$ diameter. The outer one, which generates a field in the opposite direction has 150 turns (at twice the pitch of the inner coil) with a diameter of $27.0 \mathrm{~mm}$. The coil constant of this assembly is $\mu_{0} H_{\mathrm{p}} / i_{\mathrm{p}}=3 \times 10^{-3} \mathrm{~T} \mathrm{~A}^{-1}$ and the effective self inductance is $L_{\mathrm{p}}=0.21 \mathrm{mH}$. The distance between adjacent turns is large enough that capacitive effects are negligible in the operating range of the SQUID.

The secondary coil consists of two sections of 20 turns of $0.13 \mathrm{~mm}$ niobium-titanium wire (single core, without copper matrix) with a diameter of $11.0 \mathrm{~mm}$ and a length of $3.0 \mathrm{~mm}$. The separation between the two sections is $12.5 \mathrm{~mm}$. The self in ductance $L_{\mathrm{s}}$ of each section is $6.0 \mu \mathrm{H}$ and the mutual inductance between the two is $0.9 \mu \mathrm{H}$ so that the effective self inductance of the astatic pair $L_{\mathrm{s}, \mathrm{tot}}=10.2 \mu \mathrm{H}$. The mutual inductance $M_{\mathrm{ps}}$ between the primary assembly and one section of the secondary is $5.7 \mu \mathrm{H}$. Without additional balancing the relative imbalance $\Delta M_{\mathrm{ps}} / M_{\mathrm{ps}}$ was $3.3 \times 10^{-3}$ at liquid helium temperatures.

The balancing mutual inductor has a toroidal shape and is surrounded by a lead shield to minimise its sensitivity to external magnetic fields. The secondary coil is wound on an epocast core of $9.5 \mathrm{~mm}$ diameter. It consists of 48 turns of the same wire as the sensing coils, with a diameter of $4.5 \mathrm{~mm}$. The primary coil has 48 turns of $0.1 \mathrm{~mm}$ copper wire, with a diameter of $5 \mathrm{~mm}$. The mutual inductance $M_{\mathrm{b}}$ of this assembly is $1.64 \mu \mathrm{H}$ and the primary and secondary self inductances $L_{b p}$ and $L_{\mathrm{b}}$ are $2.06 \mu \mathrm{H}$ and $1.64 \mu \mathrm{H}$, respectively. The above mentioned imbalance of the sensing mutual inductor is compensated by diverting approximately $1 \%$ of the primary current to the balancing inductor. This adjustment is independent of the temperature of the helium bath and on'y slightly dependent on the magnitude of the background field.

From the values for $L_{\mathrm{s}}, L_{\mathrm{b}}$ and $L_{\mathrm{SQ}}$ it can be calculated that the sensitivity of our system is not maximal. This reduced sensitivity even showed to be somewhat large in the experiments.

The primary coil of the balancing inductor is connected to the external feedback output of the SQUID electronics by a $24 \mathrm{k} \Omega$ resistor, which makes the effective feedback factor $\beta(\S 4)$ almost equal to the $\beta$ in the sENS $\times 1$ sensitivity setting (range $500 \varphi_{0}$ ) in the internal feedback mode.
A background field up to $5 \mathrm{~T}$ is generated by a Cryogenic Consultants 5T-60-46 superconducting magnet with a bore of $60 \mathrm{~mm}$. The persistent mode stability of this magnet is sufficient to obtain good drift-free measurements without additional field stabilisation aids. The main contribution to drifts in the input of the SQUID comes from instabilities in the temperature of the sample (certainly during experiments on a thermally isolated sample).

When the magnet is switched in the persistent mode it also provides some shielding against external magnetic fields. The noise picked up from the environment was centred mainly at the mains frequency and its odd harmonics.

Figure 6 shows the complete construction of the cryogenic part of the new susceptometer. Great care was taken to minimise the possibility of vibrations. The sensing coils, which are cast into a solid block of epoxy resin, are rigidly mounted inside the magnet. The suspension of the magnet is supported against the wall of the cryostat in order to avoid vibrations of the coils in the earth's magnetic field.

One problem, which remains to be solved, is a slight frequency dependence of the balance of the system. If there is no sample present and the balance has been adjusted at one frequency the output signal of the SQUID varies as a function of frequency as shown in figure 7 . The behaviour is almost perfectly first-order with a time constant of $8 \mathrm{~ms}$. The effect is

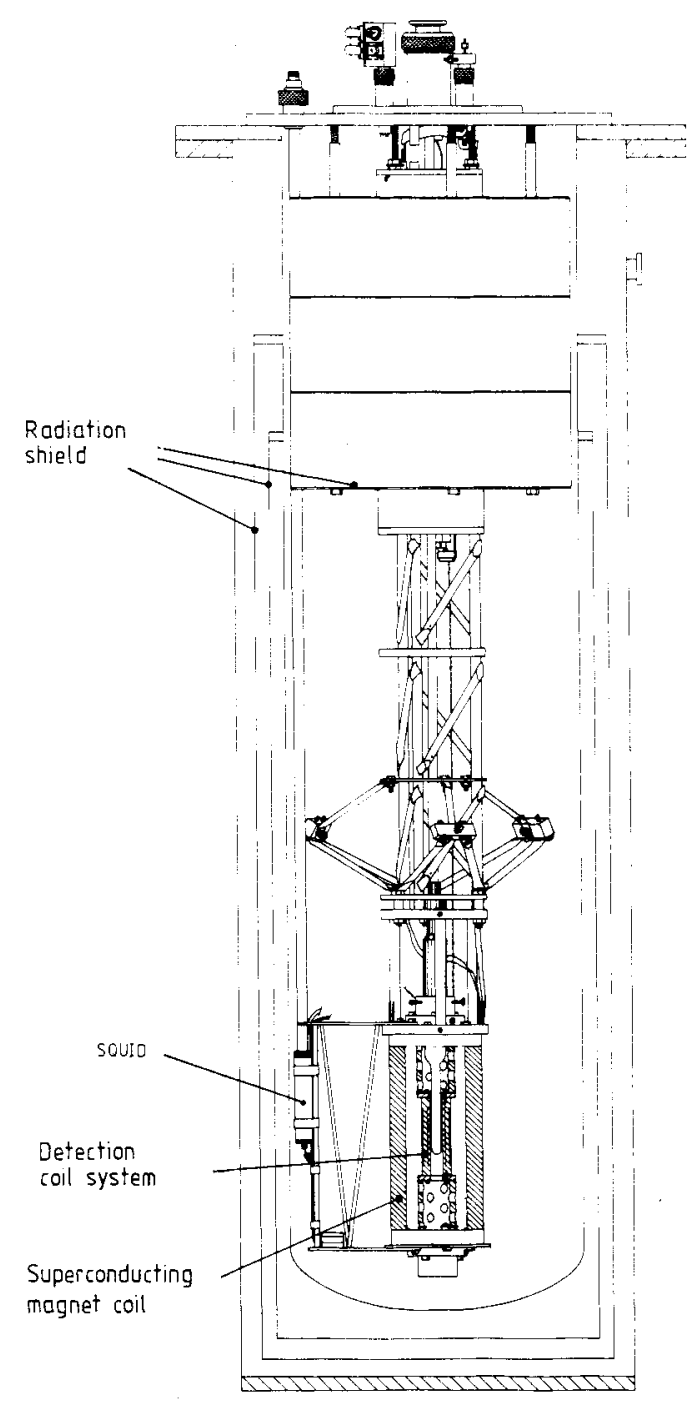

Figure 6. The low-temperature part of the experimental set-up. 


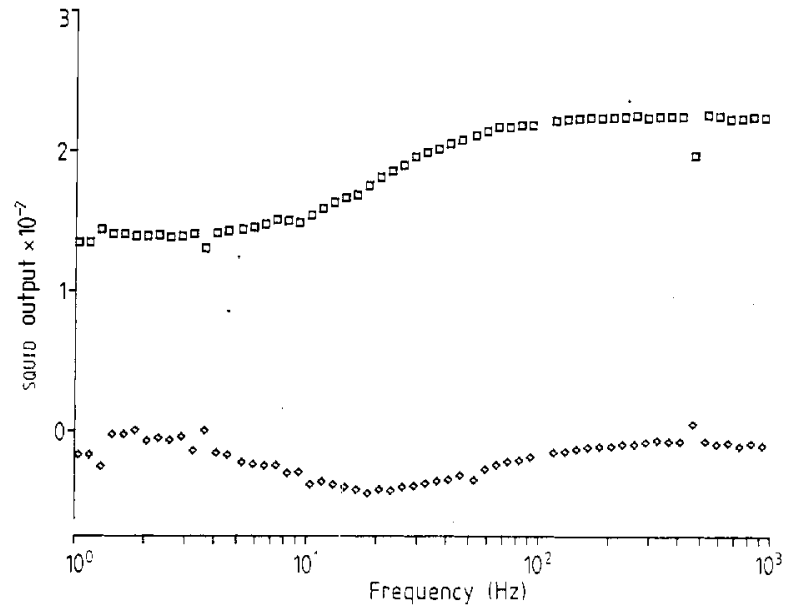

Figure 7. Empty coil response of the system. The scale for the in-phase $I(\square)$ and quadrature component $Q(\diamond)$ is according to the scale in figure 9 .
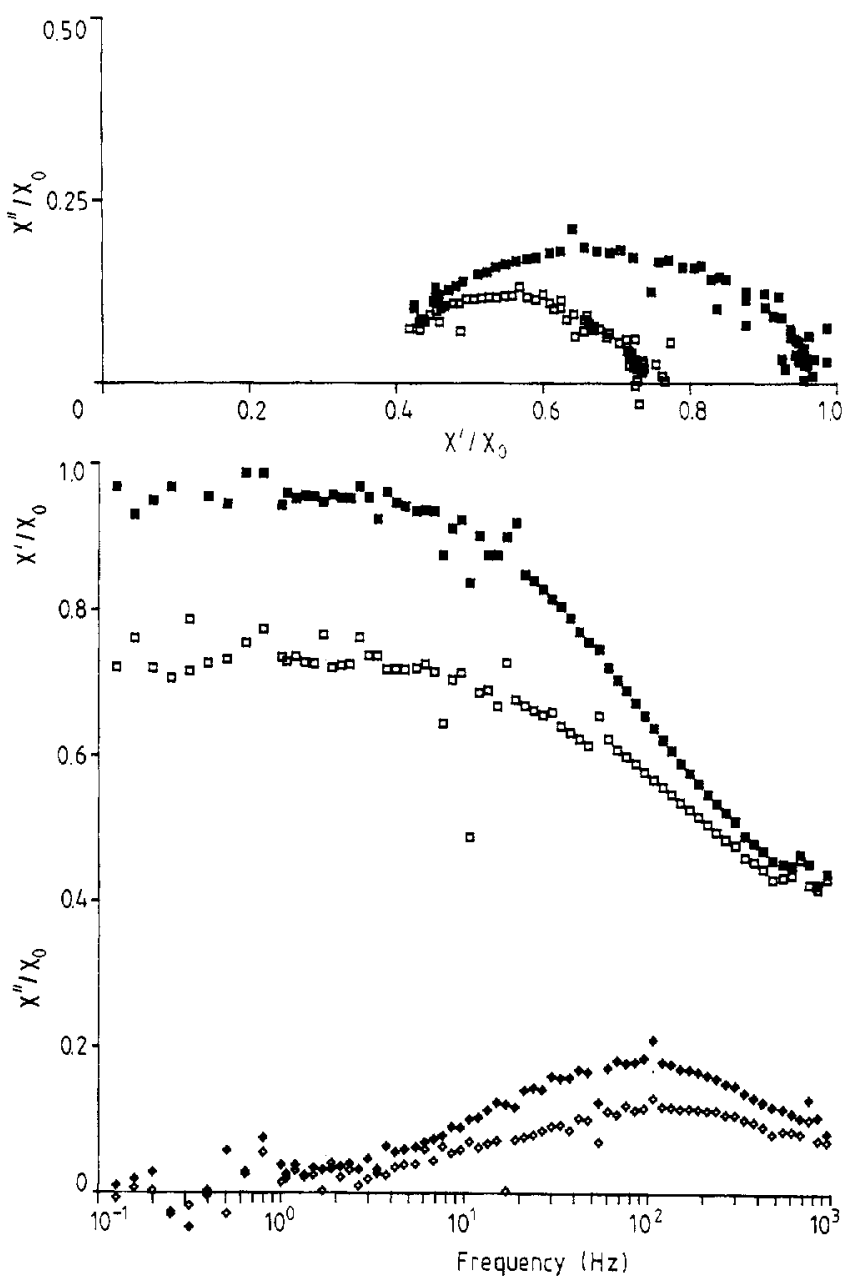

Figure 8. Argand diagram (upper figure) and Casimir-du-Pré plots (lower figure) for $\mathrm{CsMnBr}_{3} \cdot 2 \mathrm{H}_{2} \mathrm{O}$ measured with our susceptometer. $T=2.557 \mathrm{~K}, H$ parallel to easy axis and close to the spin-flop field. Full symbols, $H=1.7924 \times 10^{6} \mathrm{~A} \mathrm{~m}^{-1}$, open symbols, $H=1.7916 \times 10^{6} \mathrm{~A} \mathrm{~m}^{-1}$. linear in the primary current and completely independent of the temperature and the field. This frequency dependence is most probably caused by eddy currents induced by the small stray field of the (astatic) primary coil in the copper matrix of the conductor of the superconducting magnet. Anyway, this empty coil effect is only small (less than $5 \%$ of the signal of the potassium chromium alum sample described in $\$ 8$ ) and it is easy to correct for it by taking the difference between measurements with and without the sample. An even smaller empty coil effect was observed at very low frequencies (below $0.5 \mathrm{~Hz}$ ) at high values of the primary current. Due to the dissipation of this current the temperature of the coil system will be modulated and this causes a modulation in the magnetisation of paramagnetic impurities in the construction materials. This effect can be avoided by the use of superconducting wire for the primary coils.

After substraction of the empty coil signal, the frequency response of the complete susceptometer is nearly equal to that of the SQuid alone (figure 4 , curve $B$ ), indicating that the mutual inductors do not contribute significantly to the total phase shift of the system.

\section{Application of the susceptometer to magnetic relaxation experiments}

In this section we demonstrate our automatic frequencysweeping SQUID-based susceptometer on relaxation measurements on $\mathrm{CsMnBr}_{3} \cdot 2 \mathrm{H}_{2} \mathrm{O}$ at the spin-flop phase transition

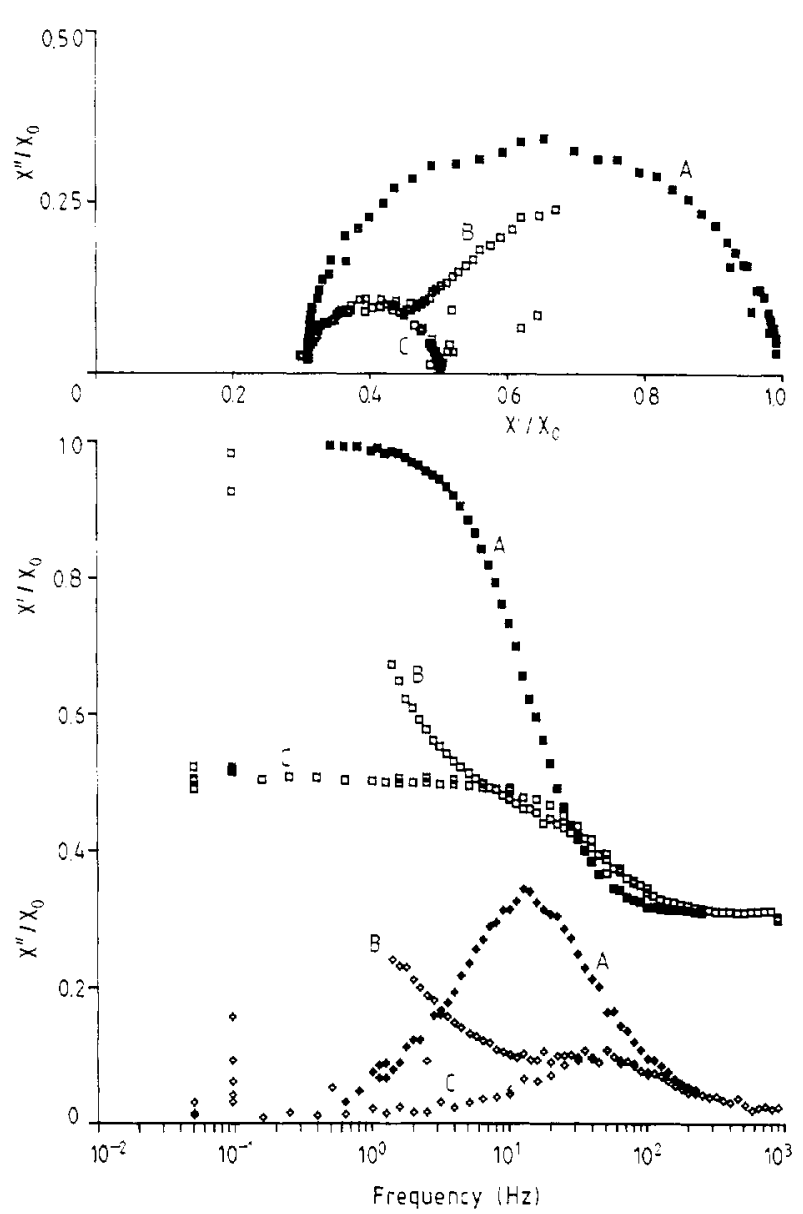

Figure 9. Argand diagram and Casimir-du-Pré plots for $\mathrm{KCr}\left(\mathrm{SO}_{4}\right)_{2} \cdot 12 \mathrm{H}_{2} \mathrm{O} . \mathrm{T}=2.267 \mathrm{~K}, H=95.8 \times 10^{3} \mathrm{~A} \mathrm{~m}^{-1}$ A, saturation pressure; $\mathrm{B}, P=10^{-2}$ Torr $(\sim 1.33 \mathrm{~Pa})$; C, $P<10^{-5} \operatorname{Torr}(\sim 1.33 \mathrm{mPa})$. 
and on $\mathrm{KCr}\left(\mathrm{SO}_{4}\right)_{2} \cdot 12 \mathrm{H}_{2} \mathrm{O}$ under various environmental conditions.

The magnitude of the susceptibility at the spin-flop transition in $\mathrm{CsMnBr}_{3} \cdot 2 \mathrm{H}_{2} \mathrm{O}$ is strongly dependent on the direction of the external field with respect to the easy axis and the transition takes place in a very narrow field interval. Using a mutual inductance technique and sweeping the field it is almost impossible to derive a reliable set of measurements at different frequencies in order to compile the Argand diagram or the Casimir-du-Pre plots. With the frequency-sweeping susceptometer the relaxation at the spin-flop field can easily be measured keeping the background field in the persistent mode. In figure 8 we present the results for sweeps at two magnetic fields in the spin-flop transition range. The total sweep time was about five minutes.

In figure 9 the results of frequency sweeps for potassium chromium alum are presented. The measurements were performed for different environmental circumstances of the single crystal (at $T=2.267 \mathrm{~K}$ ). In curve $A$ the crystal is surrounded by helium gas at the saturation pressure and thus covered by a thin helium film. In curve $B$ the pressure of the helium gas is $10^{-2}$ Torr $(\sim 1.33 \mathrm{~Pa})$ and at low frequencies the lattice-to-bath relaxation due to the contact gas is visible. The sample is thermally isolated at a helium gas pressure of $10^{-5}$ Torr $(\sim 1.33 \mathrm{mPa}$ ) (curve $C$ ). In all three cases in the figure a field-sweep method for relaxation study cannot be used owing to the magnetocaloric effect. Then one has to measure at a fixed field, point by point, and this takes an enormous length of time. With our susceptometer such measurements can be completed within a few minutes.

\section{References}

Cerdonio M, Cosmelli C, Romani G L, Messana C and Gramaccioni C 1976 Superconducting magnetometer for high resolution susceptibility measurements

Rev. Sci. Instrum. 47 1-5

Cukauskas E J, Vincent D A and Deaver B S 1973 Magnetic susceptibility measurements using a superconducting magnetometer

Rev. Sci. Instrum. 45 1-6

Flokstra J, Gerritsma G J, Kreuwel H J M and

Van der Marel L C 1980. Two high-frequency mutual inductance bridges with high resolution

J. Phys. E: Sci. Instrum. 13 1071-4

Gerritsma G J, Van Weezep W J, Overweg J A and Flokstra J $1983 \mathrm{An}$ on-line digital phase sensitive detector in the range $2 \mathrm{mHz}-2 \mathrm{kHz}$

J. Phys. E: Sci. Instrum. $16270-7$

Giffard R P, Gallop J C and Petley B W 1976 Applications of the Josephson effects

Prog. Quantum Electron. 4 301-402

Hirschkoff E C, Symko O G and Wheatley J C 1971 Magnetic behaviour of dilute $\mathrm{Cu}(\mathrm{Mn})$ alloys at very low temperatures J. Low Temp. Phys. 5 155-76

Overweg J A, Flokstra J and Gerritsma G J 1980 A SQuidbased frequency-sweeping system for AC-susceptibility measurements

Proc. ISMAR-AMPERE, Delft Bull. Magn. Res. 2 433-6

Overweg J A, Flokstra J and Gerritsma G J 1982 The influence of the environment on the dynamic susceptibility of cerium magnesium nitrate in strong magnetic fields

\section{Physica 112B 381-8}

Philo J S and Fairbank W M 1977 High-sensitivity magnetic susceptometer employing superconducting technology

Rev. Sci. Instrum. 48 1529-36
Van de Klundert L J M, de Rooij C, Caspari M and Van der Marel L C 1975 Induction methods used in low temperature physics

Cryogenics 15 577-89

Williamson S J and Kaufman L 1981 Biomagnetism

J. Magn. Magn. Mater. 22 129-201 